\title{
ALTEC y la Gestión Tecnológica en Iberoamérica: Gestión de Proyectos, Conocimiento e Innovación Social y Sustentable
}

\author{
Domingo González', Roberto Sbragia², Oscar Galante², Rocío Soto ${ }^{4}$, Renato Valdivieso ${ }^{5}$
}

\begin{abstract}
The seminars of the Latin Ibero-American Association of Technology Management (ALTEC) they are one of the most important spaces in this region for the discussion of the research in the discipline of innovation and technology management. This article presents a brief panorama of the main thematic areas approached in 14 seminars carried out by that association from 1985 until the year 20II. In this context, a group of 28 articles is presented, which discuss in this volume the project management of R\&D and innovation, the knowledge management, the social innovation and the relationships between innovation and sustainable development.
\end{abstract}

Keywords: project management, R\&D; knowledge management; social innovation.

Los encuentros bianuales realizados por la Asociación Latino-Iberoamericana de Gestión Tecnológica (ALTEC) constituyen en la actualidad uno de los espacios más importantes en esta región para la discusión de la investigación en la disciplina de la gestión de la innovación y la tecnología. En este artículo se presenta brevemente un panorama de las principales áreas temáticas abordadas en 14 encuentros realizados por esa asociación desde 1985 hasta el año 20II. En este contexto, se presenta un conjunto de 28 artículos que tratan en este volumen de la gestión de proyectos de I+D e innovación; las relaciones entre la innovación y la gestión del conocimiento, la innovación social y el desarrollo sustentable.

Keywords: gestión de proyectos, I+D, gestión del conocimiento; innovación social.

I Departamento de Ingeniería, Pontificia Universidad Católica del Perú. Av. Universitaria I80I, San Miguel, Lima 32, Perú. Telefono: +51.1.6262000. Contacto principal: dgonzal@pucp.edu.pe

${ }^{2}$ Departamento de Administração, Universidade de São Paulo. Av. Prof. Luciano Gualberto, 908, São Paulo, CEP: 05508-0 I0, Brasil.

${ }^{3}$ Instituto Nacional de Tecnología Industrial. Colectora de Avenida General Paz 5445, Casilla de correo I57, BI650KNA. San Martín, República Argentina.

${ }^{4}$ Instituto Politécnico Nacional (IPN). Av. Luis Enrique Erro S/N, Unidad Profesional Adolfo López Mateos, Zacatenco, Delegación Gustavo A. Madero, C.P. 07738, México, Distrito Federal, 2009-20I0.

${ }^{5}$ Fundación Empresas Polar. Segunda avenida, Los Cortijos de Lourdes, Edificio Fundación Empresas Polar, I Piso. Apartado postal 70934. Los Ruices. Zona postal: I07I-A. Caracas. Venezuela.

ISSN: 07 I8-2724. (http://www.jotmi.org)

Journal of Technology Management \& Innovation (c) Universidad Alberto Hurtado, Facultad de Economía y Negocios. 


\section{Introducción}

La Asociación Latino-lberoamericana de Gestión Tecnológica - ALTEC, fue fundada en octubre de 1984 con el propósito de vincular personas, físicas y jurídicas, activas en la reflexión y el ejercicio de la gestión tecnológica para realizar actividades de cooperación en dicha área. La asociación está integrada por un grupo significativo de profesionales de la región interesados en la política y gestión de la innovación y la tecnología, incluyendo entre sus más de 500 asociados a investigadores, profesores universitarios, empresarios y profesionales de las empresas, especialistas gubernamentales, expertos de agencias financieras y de cooperación y profesionales que se desempeñan en consultoras privadas.

Los orígenes de la asociación se encuentran en la Reunión Latinoamericana de Gestión de la Innovación Tecnológica promovida por la Organización de los Estados Americanos (OEA) y el Gobierno del Brasil, la cual se realizó octubre de 1983, en las instalaciones de la Universidade de São Paulo en Brasil. En ese encuentro se examinó el estado del arte sobre la innovación tecnológica en América Latina y se propusieron mecanismos y medidas para su dinamización, con énfasis en la integración del sector productivo con los esfuerzos de investigación realizados en institutos especializados de la infraestructura científica - tecnológica y en las universidades (Galante y Pérez, 2008).

La favorable experiencia de la reunión estimuló a multiplicar las observaciones, análisis y conclusiones de los diversos problemas del desarrollo científico y tecnológico de los países de la región latinoamericana. Los resultados conseguidos en este ejercicio y en otros similares indicaron la conveniencia de institucionalizar los intercambios, encuentros y colaboración entre los diversos centros de estudios y de capacitación establecidos en los países latinoamericanos, para la solución de los diversos problemas de la administración de la ciencia y tecnología. A partir de esta conclusiones, se propuso la creación de una asociación flexible integrada por personas e instituciones interesadas, a fin de enfrentar mejor la agenda del problema que comprendía cuestiones de complejidad creciente, como eran el financiamiento de la ciencia y tecnología, el avance las industrias basadas en la ciencia, el avance en la ciencia y tecnología mismas y la vinculación de las políticas y planes de la industria y tecnología en los niveles macro y microeconómico, entre otros.

Sobre estas bases, se funda la asociación y en septiembre de 1985 se realizó el Primer Seminario Latinoamericano ALTEC en la Universidade de São Paulo. En ella los socios debatieron, aprobaron los estatutos y acordaron las prioridades de la asociación durante los dos años siguientes. A partir de ese momento, cada dos años, de manera ininterrumpida, se realizan los encuentros de la asociación denominados ini- cialmente Seminarios y más adelante Congresos de Gestión Tecnológica. Con el tiempo los encuentros de ALTEC se han constituido como el evento académico más importante de la disciplina en Iberoamérica.

\section{El panorama de la Gestión Tecnológica en los encuentros de ALTEC}

Desde su creación, la asociación ALTEC ha realizado 14 encuentros académicos, los cuales se iniciaron con una estructura de seminario hasta constituirse en las últimas ediciones en congresos de gran magnitud. La Tabla I muestra la evolución de las ponencias presentadas en los encuentros, alcanzando casi 3000 ponencias en total desde 1985 hasta el año $201 \mathrm{l}$.

Desde sus orígenes, los encuentros de ALTEC han tratado los varios niveles de la gestión de la tecnología y la innovación, desde los aspectos al nivel de los proyectos y las empresas, pasando por los espacios interorganizacionales y de territorios donde confluyen diversos actores, hasta los aspectos contextuales y de construcción de entornos favorables al desarrollo de la ciencia, la tecnología y la innovación. Del mismo modo, a lo largo del tiempo, los encuentros de ALTEC han venido incorporando los nuevos enfoques $y$ conceptos y los temas de actualidad en la disciplina. La denominación de las áreas temáticas ha variado a lo largo del tiempo, sin embargo, de manera general se han abordado las grandes áreas temáticas indicadas en la Tabla 2.

\begin{tabular}{|l|l|l|l|}
\hline Año & País & Ciudad sede & $\begin{array}{l}\text { Número de ponencias } \\
\text { presentadas }\end{array}$ \\
\hline 1985 & Brasil & Sao Paulo & 26 \\
\hline 1987 & México & México D.F. & 76 \\
\hline 1989 & Argentina & Buenos Aires & 92 \\
\hline 1991 & Venezuela & Caracas & 84 \\
\hline 1993 & Colombia & Bogotá & 53 \\
\hline 1995 & Chile & Concepción & 102 \\
\hline 1997 & Cuba & La Habana & 193 \\
\hline 1999 & España & Valencia & 256 \\
\hline 2001 & Costa Rica & San José & 196 \\
\hline 2003 & México & México D.F. & 274 \\
\hline 2005 & Brasil & Salvador & 392 \\
\hline 2007 & Argentina & Buenos Aires & 348 \\
\hline 2009 & Colombia & Cartagena & 327 \\
\hline 2011 & Perú & Lima & 514 \\
\hline & & Total & 2933 \\
\hline
\end{tabular}

Tabla I. Encuentros ALTEC 1985 - 201 I.

Fuente:ALTEC (1985; 1987; 1989; 1991; 1993; 1995; 1997; 1999; $200 I ; 2003 ; 2005 ; 2007 ; 2009 ; 201$ I)

ISSN: 07 I8-2724. (http://www.jotmi.org)

Journal of Technology Management \& Innovation (c) Universidad Alberto Hurtado, Facultad de Economía y Negocios. 
Algunas de las áreas temáticas abordadas en los catorce encuentros se han mantenido, indicando el permanente interés en la región por los temas vinculados al entorno de los sistemas de innovación y a la construcción de ambientes favorables para el desarrollo de las ciencia, la tecnología y la innovación; a la gestión de la tecnología y la innovación en la empresa; a la política científica y tecnológica y a la gestión de la I+D. Del mismo modo, se observa la emergencia de temas específicos tales como la dinámica de la creación de empresas innovadoras, la gestión ambiental y del conocimiento, entre otros. La Tabla 4 muestra el porcentaje de ponencias presentadas desde 1985 hasta 201 I en cada área temática, indicando el grado de interés en la región en cada tema.
Quirino et al. (200I), Sbragia et al. (2003) han estudiado en años pasados las características de los encuentros ALTEC en los temas y número de trabajos presentados, sistema de autoría, distribución de las ponencias por país, por instituciones y por autores con mayor contribución de ponencias a los encuentros. En aquella época los autores vislumbraron las tendencias hoy consolidadas, tales como el creciente interés en la disciplina, las preferencias por las áreas temáticas indicadas anteriormente $y$ el aumento de la coautoría en las ponencias presentadas en los encuentros.

\begin{tabular}{|c|c|}
\hline Áreas temáticas & Contenido \\
\hline $\begin{array}{l}\text { La Vinculación entre los acto- } \\
\text { res para la innovación: }\end{array}$ & $\begin{array}{l}\text { Las relaciones entre las actividades de I+D de la academia y el sector productivo; la relación universidad-empresa; } \\
\text { las estructuras organizacionales de interfaz; cooperación de la industria con entidades del estado; la transferencia } \\
\text { de los resultados de la I+D. }\end{array}$ \\
\hline $\begin{array}{l}\text { Gestión de la innovación y la } \\
\text { tecnológica en la empresa }\end{array}$ & $\begin{array}{l}\text { Gestión en empresas innovadoras; experiencias de gestión de la tecnología en sectores específicos; gestión de } \\
\text { la innovación de la micro, pequeña y mediana empresa; alianzas y cooperación interempresarial en proyectos de } \\
\text { innovación, nuevas tecnologías de producción y sistemas de información; cultura organizacional para la innovación; } \\
\text { planeamiento estratégico de la tecnología; perfil de los gestores de innovación; propiedad industrial e intelectual; } \\
\text { prospectiva y vigilancia tecnológica; gestión tecnológica en nuevos modelos de organización empresarial. }\end{array}$ \\
\hline Sistemas de innovación & $\begin{array}{l}\text { Sistemas sectoriales, regionales, nacionales y locales de innovación; redes de investigación e innovación; la } \\
\text { construcción de entornos favorables para la innovación; organización regional, nacional y estatal para la gestión } \\
\text { en ciencia y tecnología; estrategias locales y regionales de innovación tecnológica; clusters, arreglos productivos, } \\
\text { territorio y desarrollo local, territorio e industria creativa; competitividad sistémica, industrial y empresarial; } \\
\text { competitividad, empleo y desarrollo sostenible; normas técnicas y otros aspectos de la regulación de la ciencia y la } \\
\text { tecnología. }\end{array}$ \\
\hline Gestión de la I+D & $\begin{array}{l}\text { La función de I+D en la empresa; gestión de riesgo y técnicas de planeamiento, control y evaluación; organización } \\
\text { de equipos de proyectos; portafolio de proyectos; instrumentos de promoción de la I+D; política y gestión de } \\
\text { investigación e innovación tecnológica en universidades e institutos de investigación; evaluación del desempeño en } \\
\text { instituciones de investigación. }\end{array}$ \\
\hline Política científica y tecnológica & $\begin{array}{l}\text { Políticas, fuentes e instrumentos de financiamiento de la innovación tecnológica; aspectos jurídicos de la inno- } \\
\text { vación; política de innovación: programas y redes internacionales; Integración latinoamericana y gestión tecnológi- } \\
\text { ca; incentivos fiscales, no fiscales y otros mecanismos inductores de innovación; los impactos sociales, económicos } \\
\text { y ambientales de la innovación; indicadores de ciencia, tecnología e innovación; evaluación en I+D e innovación. }\end{array}$ \\
\hline $\begin{array}{l}\text { Creación de empresas innova- } \\
\text { doras y de base tecnológica, } \\
\text { incubadoras, polos y parques }\end{array}$ & $\begin{array}{l}\text { Emprendedores y generación de empresas de base tecnológica; financiamiento de nuevas empresas; planeamiento } \\
\text { y gestión de incubadoras, polos y parques tecnológicos. }\end{array}$ \\
\hline Gestión del Conocimiento & $\begin{array}{l}\text { La innovación y la gestión del conocimiento; generación, gestión y transferencia del conocimiento; indicadores y } \\
\text { gestión del conocimiento; gestión del conocimiento y de la información, calidad y productividad. }\end{array}$ \\
\hline Ciencia,Tecnología y Sociedad & $\begin{array}{l}\text { Aspectos sociales y culturales de la gestión tecnológica; innovación, ética y sociedad; economía solidaria e inno- } \\
\text { vación tecnológica; estudios de género y tecnología; tecnología y gestión urbana; tecnología social; innovación } \\
\text { social. }\end{array}$ \\
\hline Formación de capacidades & $\begin{array}{l}\text { Formación de recursos humanos en gestión tecnológica; formación para el desarrollo de la capacidad innovadora; } \\
\text { Tecnología, formación y empleabilidad; estrategias de aprendizaje y construcción de capacidades de innovación. }\end{array}$ \\
\hline $\begin{array}{l}\text { Desarrollo sustentable y } \\
\text { gestión ambiental }\end{array}$ & $\begin{array}{l}\text { Relaciones entre desarrollo tecnológico y medio ambiente; aspectos ambientales de la innovación tecnológica; } \\
\text { Gestión tecnológica para el desarrollo sustentable; gestión ambiental. }\end{array}$ \\
\hline $\begin{array}{l}\text { Herramientas de apoyo a la } \\
\text { gestión de la I+D e innovación }\end{array}$ & $\begin{array}{l}\text { Aplicación de técnicas específicas cualitativas y cuantitativas; propuesta, desarrollo y aplicación de métodos y } \\
\text { metodologías para la gestión de la I+D e innovación, }\end{array}$ \\
\hline Temas emergentes & $\begin{array}{l}\text { Nuevos desarrollos y conceptos en política de ciencia tecnología e innovación; nuevos conceptos, metodologías y } \\
\text { técnicas en la gestión de la innovación y la tecnología. }\end{array}$ \\
\hline
\end{tabular}

Tabla 2. Áreas temáticas de los encuentros ALTEC.

Fuente:ALTEC (1985; 1987; 1989; I99I; 1993; 1995; 1997; 1999; 200I; 2003; 2005; 2007; 2009; 20I I)

ISSN: 07 I8-2724. (http://www.jotmi.org)

Journal of Technology Management \& Innovation (c) Universidad Alberto Hurtado, Facultad de Economía y Negocios. 


\section{La Edición Especial}

En este volumen se presentan 28 artículos que abordan tres áreas de interés para la política y gestión de la tecnología en la región latinoamericana: la gestión de proyectos de I+D e innovación; las relaciones entre la innovación y la gestión del conocimiento y; la innovación social y el desarrollo sustentable.

En la primera área temática se presentan cuatro artículos que indagan sobre cómo se está realizando la gestión de proyectos en casos específicos. Los proyectos son considerados la célula-mater de la innovación, a través de los cuales las innovaciones ocurren en el contexto organizacional, desde el punto de vista estratégico hasta el operacional, pasando por el táctico. Desde el punto de vista estratégico, las organizaciones requieren seleccionar los proyectos adecuados para invertir, a partir del enfoque de la gestión de portafolio y de las métricas de evaluación de éxito. Desde el punto de vista táctico, es necesario crear y sostener una gobernanza orientada a proyectos, donde se destaca la autonomía de los gerentes en sus articulaciones con las estructuras funcionales. Finalmente, del punto de vista operacional, son necesarios procesos y prácticas gerenciales que garanticen la eficiencia y eficacia del ciclo de desarrollo de nuevos productos, procesos y servicios.

Desde el punto de vista estratégico, los trabajos de Moraes y Laurindo (2012a y 20I2b) desarrollan un conjunto de referencias y métricas para la evaluación de la madurez organizacional y del desempeño de proyectos, partiendo de modelos teóricos recientes y terminando con el análisis de los resultados de encuestas aplicadas a varias empresas bra- sileñas del sector de tecnologías de la información. Por otro lado, los trabajos de Rodrigues (2012) y Rabechini y Carvalho (20I2) abordan la gestión de proyectos desde el punto de vista operacional, enfocándose en algunos procesos de gestión. Así, Rodrigues (20/2) analiza la influencia de la diversidad cultural e las prácticas de la gestión de equipos globales en algunas empresas brasileñas internacionalizadas. Finalmente, Rabechini y Carvalho (20I2), analizan los impactos de las prácticas de gestión del riesgo sobre el éxito de proyectos a partir de una extensa investigación de campo también en el contexto brasileiro.

La innovación debe ser vista como un fenómeno complejo que involucra diferentes posibilidades de inversión. Desde esta perspectiva, el conocimiento debe ser considerado como una especie de capital. De hecho, el éxito de una estrategia de innovación está fuertemente conectado con el trabajo de I+D, por consiguiente, de los niveles de conocimiento existentes y del esfuerzo de los colaboradores para buscar y utilizarlo creativamente. De acuerdo con los enfoques recientes, la gestión del conocimiento es un factor clave de éxito porque trata con recursos que pueden ayudar a los tomadores de decisión a evaluar situaciones y definir estrategias vencedoras.

En este sentido, en el área temática de la gestión del conocimiento se presentan seis artículos que abordan ese fenómeno, desde diferentes ángulos. Los trabajos de Ferreira y Pilatti (20I2), Agrasso Neto (20I2) y Hernández y Castellanos (20I2) se ubican en una perspectiva más general. Por un lado, Ferreira y Pilatti (20I2), abordan cómo la gestión del conocimiento, en sus diferentes dimensiones, es percibida en las organizaciones investigadas, a partir de una encuesta

\begin{tabular}{|l|l|}
\hline Área Temática & Ponencias (\%) \\
\hline Sistemas de innovación & 19.8 \\
\hline Gestión tecnológica en la empresa & 19.7 \\
\hline Vinculación entre los actores & $\mathrm{Il} .4$ \\
\hline Gestión del conocimiento & $\mathrm{I}$ I.I \\
\hline Creación de empresas innovadoras y de base tecnológica, incubadoras, polos y parques & 7.1 \\
\hline Gestión de la I+D & 6.3 \\
\hline Ciencia, tecnología y sociedad & 6.0 \\
\hline Política científica y tecnológica & 5.2 \\
\hline Herramientas de apoyo a la gestión de la I+D e innovación & 4.9 \\
\hline Desarrollo sustentable y gestión ambiental & 4.2 \\
\hline Formación de capacidades & 3.0 \\
\hline Temas emergentes & 1.3 \\
\hline Total & 100.0 \\
\hline
\end{tabular}

Tabla 4. Ponencias por áreas temáticas de los encuentros ALTEC (I $985-201$ I) Fuente:ALTEC (1985; 1987; 1989; 199I; 1993; 1995; 1997; 1999; 2001; 2003; 2005; 2007; 2009; 20I I)

ISSN: 07 I8-2724. (http://www.jotmi.org)

Journal of Technology Management \& Innovation (c) Universidad Alberto Hurtado, Facultad de Economía y Negocios. 
en una muestra de colaboradores. Por otro lado, Agrasso Neto (20I2) analiza las mejores prácticas de inteligencia competitiva y gestión del conocimiento en empresas brasileñas mediante un enfoque descriptivo. Finalmente, Hernández y Castellanos (20I2), a partir de un estudio bibliométrico, discuten el valor de la tecnología tomando en cuenta el carácter intangible del conocimiento incorporado en la misma, contrastando con enfoques tradicionales de valorización, como el financiero.

Los trabajos de Caldas y Candido (20I2), Ress, Moraes y Salerno (20I2) y Godói-de-Sousa y Nakata (20I2). Son más específicos en su enfoque de la gestión del conocimiento. Inicialmente, Caldas y Candido (2012) analizan la dinámica de la conversión del conocimiento en espacios $\mathrm{Ba}$ (espacios físicos, mentales y virtuales) existentes en redes de colaboración interorganizacional. Por otro lado, Ress, Moraes y Salerno (20/2) discuten la técnica del TDD- Test Driven Development como proceso de mejoramiento del trabajo en equipos de desarrollo cuando ocurre una eficaz conversión del conocimiento entre clientes y desarrolladores. Finalmente, Godói-de-Sousa y Nakata (20I2) analizan las comunidades de práctica como forma de desarrollar el conocimiento, trabajando algunos de sus condicionantes.

En el área temática innovación social se presentan 9 trabajos que abordan asuntos relacionados a la responsabilidad social empresarial; a las tecnologías sociales; al emprendedorismo social; al uso de las redes sociales innovadoras, a la transferencia de tecnología a emprendimientos cooperativos; a metodologías de planeamiento de parques de innovación y servicios y al estudio del marco legal de la diseminación de tecnologías.

El primer trabajo de Penteado et al. (2012) aborda la dimensión ética de la Responsabilidad Social Empresarial (RSE) y cómo este tema ha ido creciendo, tanto en los ámbitos académicos, como en los empresariales y gubernamentales. Específicamente, la investigación se enfoca el sector académico y a partir de una encuesta, se indaga entre los alumnos de ingeniería y tecnología de la Universidad Tecnológica Federal de Paraná alumnos sobre sus percepciones respecto a la responsabilidad social en las empresas, en la universidad y sobre sí mismos. Los autores debaten sobre la RSE, a nivel ético, legal, ambiental y socio-económico. Entre las conclusiones obtenidas, sobresale el hecho de que los alumnos perciben que las empresas se deben responsabilizar de resolver los problemas sociales que ellas mismas generan, en función de mantener su imagen de desarrollo sustentable. Con relación al sector académico, los autores analizan que los alumnos perciben que este tema los posiciona socialmente a nivel profesional, y en lo personal los alumnos son conscientes que las mejores prácticas de RSE son aquellas que preservan el medio ambiente, la diversidad, las que tienden a disminuir las desigualdades sociales $y$ las que promueven que se respeten los derechos humanos.

Por otro lado, Cazini (2012) trata sobre las innovaciones tecnológicas sociales y como estas facilitan mejoras en la calidad de vida de la sociedad y fundamentalmente en aquellas personas con alguna capacidad diferente, permitiéndoles una mayor inclusión en el ámbito laboral. El autor analiza específicamente el uso de las tecnologías de información y comunicación, a través de la inclusión digital, en aquellos que presenten alguna deficiencia visual. La investigación es un estudio de caso del Proyecto VOZES, desarrollado entre la Universidad y una organización no gubernamental; donde a partir de cursos de informática, confirman que la inclusión digital contribuye a que esas personas con distintas capacidades, logren ser mejor incluidas en el mundo del trabajo. El proyecto utiliza para esto: teclados, impresoras en Braile, audífonos y lectores de voz, junto a un software especial desarrollado para tal efecto. Adicionalmente, en el trabajo se analiza entre otras, la producción de un material didáctico específico y la creación de un ambiente digital y una cultura inclusiva. Para esto, los autores también promueven y comprueban la influencia del impulso del Estado para lograr los objetivos previstos.

En el ámbito del emprendedorismo social, Ladeira y Machado (20/2) presentan como este fenómeno crece con fuerza en muchos países y específicamente en Brasil. A partir de esta aseveración los autores plantean la creación de instrumentos o herramientas que permitan modificar la exclusión social. Estudian entonces políticas públicas de apoyo al emprendedorismo social en Inglaterra, USA, Dinamarca, Israel y Hong Kong y discuten su aplicabilidad a la realidad brasileña. Así, los autores enfatizan el desarrollo de este tipo de emprendedorismo como valor social, por sobre los posibles retornos financieros o la maximización del lucro. Luego, los autores definen las distintas formas del emprendedorismo social concluyendo que en Brasil existen altas tasas de emprendedorismo por necesidad (compromiso por su sobrevivencia) y bajas tasas de emprendedorismo social (con objetivos comunitarios). Los autores analizan, entre otras, la necesidad de cooperación entre los Estados y el Tercer Sector; $y$ el hecho de que en sociedades desarrolladas existe una fuerte relación entre emprendedorismo y política, disminuyendo mucho esta relación en países en desarrollo. Finalmente, los autores plantean que en Brasil faltan reglamentaciones, investigaciones y apoyo gubernamental, proponiendo la creación de una agencia que centralice las políticas de apoyo al emprendedorismo social; $y$ de un banco de datos estadísticos e indicadores socioeconómicos; relevar al Tercer Sector; incentivar el estudio de la realidad local en las escuelas; hacer proyectos asociativos y organizar un sistema de apoyo financiero, entre otros puntos. 
También, Godói-de-Sousa y Valadão Junior (2012) abordan el tema del emprendedorismo social en una investigación sobre el conocimiento producido por la sociedad, promoviendo innovación social; como una práctica en construcción de asociativismo productivo. Es una investigación de carácter exploratorio, desarrollada en dos etapas, en la primera, los autores analizan un grupo de casi 400 emprendimientos solidarios, relevados por la Secretaría Nacional de Economía Solidaria asociado con el Forum Brasileño de Economía Solidaria. En la segunda etapa, sobre una muestra de $32 \mathrm{em}-$ prendimientos realizan entrevistas a los gestores principales de cada uno de ellos. Los autores analizaron las principales características de los emprendimientos sociales, el proceso de generación de conocimiento social, la estructura, el planeamiento, la comunicación, el control y evaluación de los emprendimientos investigados. La investigación plantea que el emprendedorismo social mejora la vida de la comunidad y son solidarios con los procesos de emancipación de las sociedades. Asimismo, se proponen acciones por parte de los gobiernos como capacitación, concientización y prácticas asociativas. Los autores concluyen que es necesario el asociativismo, privilegiando una dinámica de aprendizaje, a partir de la socialización de vivencias colectivas.

En el ámbito de las tecnologías sociales, Costa et al. (20I2) abordan un caso de degradación ambiental en el nordeste de Brasil, con características semiáridas y estudian acciones para racionalizar el uso de la tierra, $y$ de los recursos naturales, aumentando la productividad agrícola y garantizando la sustentabilidad ambiental, social, cultural, territorial, económica y política. Los autores destacan al Movimiento de Tecnología Social que promueve la apropiación de tecnologías simples y de bajo costo que benefician a toda la comunidad, y el desarrollo sustentable en el interior del Estado de Ceará. Como política pública de ese estado se desarrolla el Proyecto Mandalla, en el marco del cual los autores estudian, a través de una investigación de campo, alrededor de 150 proyectos implementados a lo largo de un año, con participación de casi 500 productores. El Proyecto Mandalla utiliza un sistema de producción agropecuaria con irrigación y crianza de pequeños animales, para generar transformaciones sociales a partir de la democratización del conocimiento. Los resultados del estudio muestran que los proyectos implementados proporcionan muchas contribuciones a los agricultores familiares y a la sociedad, concluyendo este tipo de tecnologías sociales mejoran la efectividad de las políticas públicas.

Por otro lado, Medeiros et al. (20I2) abordan la utilización de las redes sociales y su impacto como espacio organizacional; considerando el dominio del tiempo, el espacio y el capital, que repercute en las relaciones sociales. Los autores consideran que el avance tecnológico genera nuevos conceptos de tiempo y espacio, más allá de la presencia física.
En este marco, anotan que las Tecnologías de la Información (TI) permiten interacciones virtuales y simbólicas y posibilitan transformar el espacio organizacional. La investigación analiza la aplicación de las TI en compañías bien posicionadas y a través de un abordaje cualitativo, muestran que el uso corporativo de las redes sociales viabiliza la redefinición y ampliación del espacio organizacional. Así, los autores plantean la utilización y potenciación de nuevos espacios que permiten asociadamente la interacción entre distintos tipos de usuarios, consumidores, instituciones y funcionarios. Los autores identifican las redes sociales que emplean estas empresas y analizan el uso dado, concluyendo que el fenómeno de las redes sociales en línea, ha modificado el comportamiento de individuos, grupos y organizaciones, ampliando el espacio organizacional de las empresas estudiadas.

Guagliano et al. (2012) describen una experiencia piloto de transferencia de tecnología solar en Argentina, con la particularidad que esta transferencia se realiza desde un centro de investigación y desarrollo público a una cooperativa de trabajadores en una provincia norteña, para que puedan producir cocinas solares, convirtiéndose en una innovación social y de mercado en esa región. Los autores analizan también la participación de varios actores para que la transferencia, ya que la experiencia también contó con el apoyo financiero, técnico y político de dos ministerios a nivel nacional: el de Desarrollo Social y el de Ciencia, Tecnología e Innovación Productiva, además del gobierno Municipal y de un Centro de Investigaciones del CONICET de Argentina. En el trabajo se describe la experiencia, los procesos técnicos, económicos y comerciales que intervienen; articulando con un marco teórico que permite comprender el significado de la experiencia y los condicionantes que existieron. La investigación destaca la cooperación intrainstitucional en el proyecto, como así también el trabajo en equipos interdisciplinarios, que generaron aprendizajes cruzados entre todos los actores intervinientes y la construcción de consensos para el logro de futuros posibles y deseables.

Melo y Magacho (20I2) plantean identificar metodologías adecuadas para planear un Parque de Innovación y Servicios (PISP), en función de lograr un fomento al desarrollo local, humano y sustentable. En ese marco se promueve la cooperación interuniversitaria $y$, sobre todo, un nuevo concepto de Universidad que, aparte de generadora, transmisora y aplicadora de conocimientos; busque, proponga y desarrolle la innovación y el emprendedorismo. Las autoras entienden que de esta manera se mejora la calidad de vida y el bienestar social, fomentando disminuir las desigualdades sociales. También, el artículo analiza el concepto de la Triple Hélice e introduce a los ciudadanos como agentes complementarios; articulando múltiples actores en el complejo proceso de planificación adaptativa, considerando la especificidad en relación a otro tipo de parques científicos y tecnológicos. 
En el artículo se introduce el concepto de dominio interorganizacional, destacando la heterogeneidad de los actores y las exigencias de capacitación e infraestructura. De esta manera, coinciden en que los resultados de este tipo de trabajo, pueden servir de base para la implementación de políticas públicas tendientes a apoyar la generación de proyectos en ambientes innovadores.

Rodrigues, Lage y Vasconcellos (20I2) abordan la conexión entre la evolución de los marcos legales relacionados al cultivo de la soja y el crecimiento del cultivo de soja transgénica en el territorio brasileño, de forma a determinar el rol de esa soja en el escenario actual de la agricultura nacional. El artículo analiza las leyes en ese país de Propiedad Industrial, de Protección de Cultivo y la de Semillas; y establecen sus diferencias y sus interfaces. También, analizan la Ley de Bioseguridad. Con datos del Ministerio de Agricultura, estiman el uso de semillas tolerantes al herbicida frente a las no transgénicas en el Brasil. Finalmente, los autores plantean la necesidad de que el Estado regule el mercado de semillas a través de la adopción de políticas públicas que apliquen las leyes actuales, aumentando la fiscalización e incentivando la I+D de los sectores público y privado.

El último tema de este volumen trata sobre la innovación y el desarrollo sustentable. El desarrollo de modelos de innovación tecnológica sustentables orientados al logro de una mayor racionalización del uso de los recursos naturales, una mayor eficiencia productiva por la vía de la disminución del consumo de materias primas, energía, agua y hacia la reducción y reaprovechamiento de los residuos, constituye uno de los desafíos más importantes de la humanidad en este milenio que apenas comienza.

Cada día hay más consciencia y crece la comprensión sobre la necesidad de cuidar el medio ambiente y sobre las acciones en el ámbito de la producción industrial y de las normativas globales que es necesario desarrollar, con amplios criterios de inclusión, para la preservación de la vida en el planeta. Este camino ha sido posible transitarlo gracias a esfuerzos pioneros como el realizado por la Comisión Brundtland en 1987, que puso en el tapete el concepto de desarrollo sustentable o los pasos de avance dados por buena parte de los países del mundo con su adhesión al Protocolo de Kyoto en 2005 y la determinación de metas para la reducción de las emisiones de gases de efecto invernadero. En este sentido, los ojos del planeta han volteado hacia el bosque amazónico, el llamado pulmón del mundo, para garantizar su condición de sumidero de carbono y preservar el ecosistema amazónico, fuente de una inigualable diversidad genética que genera grandes oportunidades de bioprospección, sobre todo si se incorporan los conocimientos de la población local.
Todos estos elementos justifican la importancia que al tema de la innovación tecnológica sustentable le asigna ALTEC en sus seminarios bianuales, en los cuales se aborda el tema desde múltiples perspectivas, desde los estudios prospectivos, pasando por el análisis estratégico de nuevos negocios sustentables a partir los recursos naturales o la inversión gubernamental para el estímulo a la innovación en la amazonia brasilera o en los servicios públicos. En este contexto se presenta a continuación nueve artículos sobre esta temática. Almeida y Caldas de Moraes (20I I) muestran una síntesis de los principales resultados de un interesante estudio prospectivo, adelantado por ellos en 2010 , donde se describen las trayectorias evolutivas de ocho sectores industriales considerados de gran impacto en Brasil para alcanzar un futuro sustentable, en un horizonte de veinte años. El estudio enfatiza en el entendimiento y comprensión de las fuerzas motrices, tendencias e incertidumbres críticas que condicionarán la difusión de las tecnologías emergentes (basados en los avances de la biotecnología, nanotecnología, tecnologías de la información y la comunicación y en química verde) en la configuración de las industrias existentes en ese país y en futuras industrias en otros sectores considerados. Igualmente, se toma muy en cuenta en el análisis el posicionamiento estratégico de Brasil frente a las trayectorias globales de los sectores considerados y el debido aprovechamiento de las oportunidades tecnológicas y de negocios que se vislumbran.

Frikman y Vasconcellos (20II) analizan el impacto de las inversiones públicas para el desarrollo de la ciencia, tecnología e innovación en la amazonia brasilera usando como indicador de la actividad innovadora el número de solicitudes de patentes depositadas en el Instituto de Propiedad Industrial (INP). Según los autores, a pesar del incremento del financiamiento público y del apoyo político recibido para estimular la investigación sobre la biodiversidad amazónica, los resultados son aún escasos en el campo de los bioproductos que utilizan recursos vegetales de la región, si se comparan, por ejemplo, con otros sectores como el electro electrónico. No obstante, consideran fundamental la permanencia de los estímulos fiscales y la atracción de medianas y grandes empresas que aprovechen los insumos naturales $y$ que puedan intercambiar experiencias y conocimientos con las micro y pequeñas industrias regionales, estimulando así la organización y crecimiento de cadenas productivas regionales basadas en las actividades extractivas tradicionales. Estos procesos deben ser, de acuerdo con los autores, regidos por fuertes estrategias de gobernanza, capaces de regular las actividades empresariales sin que ellas impidan el desarrollo de las mismas. De esta forma, los autores señalan que el éxito de la innovación biotecnológica podrá compararse con el de otros sectores en los próximos 30 años, garantizando así otro frente de desarrollo sustentable para la amazonia brasilera en el siglo XXI. 
Partiendo de la idea de que cada vez más la competitividad y la inserción internacional de las empresas brasileras dependen de la inversión en las actividades de innovación tecnológica y de desarrollo sustentable, el estudio de Gomes et. al. (20I I) tiene por objeto identificar las principales estrategias de negocios sustentables y los indicadores que reflejan el desempeño empresarial de las empresas estudiadas. Participaron en el estudio 93 empresas asociadas a ANPEI, a las que se les hizo una encuesta con base a un modelo conceptual que relaciona la variable gestión para el desarrollo sustentable con la variable desempeño empresarial. Los resultados muestran como las empresas brasileras están ampliando su participación en los mercados internacionales, no obstante, observan algunos factores que limitan esa actuación en términos de gestión de innovación y de gestión socio ambiental. En lo referente a la gestión de la innovación tecnológica los autores sostienen que hará falta desarrollar una cultura empresarial que valorice las alianzas y la colaboración entre empresas y que las estructuras de las empresas deberán ser trabajas para favorecer la gestión de proyectos conjuntos y destacan el rol del poder público como elemento de articulación que favorece el desarrollo de estos procesos.

En cuanto a la gestión socio ambiental la investigación corrobora lo reportado en la literatura que afirma que las empresas brasileras adoptan una postura reactiva frente a estas cuestiones, orientando sus inversiones y acciones para atender y cumplir las exigencias de las normas nacionales e internacionales. Gomes et. al. (20II) concluyen afirmando que "un cambio significativo de esta postura será necesario en procura de mayores niveles de competitividad empresarial, lo cual pasa por el convencimiento de que la inversión en innovación, comprometida con acciones sustentables, constituye una manera concreta de agregar valor al producto nacional y superar las barreras y condicionantes de actuación y competitividad internacional."

El objetivo principal del estudio de Menezes et. al. (20I I) es identificar las principales formas de gestión de la innovación tecnológica sustentable en la industria química brasilera y verificar la relación de la adopción de estas prácticas con el incremento de los procesos de inserción internacional de dicha industria. Los autores justifican la selección de este sector industrial tanto por su alto potencial de generación de innovación tecnológica como de residuos y contaminantes asociados a sus productos y procesos productivos. Igualmente, es un sector muy importante dentro del conjunto de la industria brasilera, tanto por su un alto nivel de producción, como por su participación internacional, así como también por su intervención en casi todas las cadenas y complejos industriales del país.
Para llevar adelante la investigación se seleccionó una empresa química localizada en el sur de Brasil con una actuación destacada en innovación y una relevante participación en los mercados internacionales. El estudio de caso, de carácter descriptivo y cualitativo, fue efectuado a partir de entrevistas semi-estructuradas con los responsables de la gestión tecnológica. El modelo conceptual utilizado revela dos dimensiones, "gestión de la innovación tecnológica" y "performance internacional". Los resultados del análisis de la información recabada muestran que las prácticas de gestión de la innovación en productos y procesos sustentable en la empresas se caracteriza por la creciente inversión en innovaciones en productos y procesos, resultado de alianzas diversas con centros de I\&D nacionales y con empresas foráneas que, según los autores, promueven el desarrollo de nuevos productos y la ampliación del mercado de la empresas. La hipótesis sugerida por los autores que propone una relación entre la gestión de innovación tecnológica sustentable y el incremento de los procesos de inserción internacional del sector químico, para el caso de la empresa estudiada, no fueron concluyentes. Ellos sostienen que los datos evidencian que las prácticas de gestión de la innovación sustentable pueden constituirse en aspectos motivadores para la búsqueda de alianzas internacionales y de innovaciones que podrán transformarse en oportunidades de negocios para el mercado doméstico e internacional.

De acuerdo con Tonelli et. al. (20II) uno de los grandes desafíos de la empresa en la actualidad es producir teniendo en cuenta la capacidad de carga del ecosistema ambiental donde opera. Los autores sostienen que son innúmeras las iniciativas de creación de alternativas de producción no agresivas con el medio ambiente capaces de articular la producción de riquezas y el bien común de la sociedad, por eso, el objetivo que se plantean en esta investigación es demostrar si estas tendencias de producción se inscriben en una perspectiva de innovación sustentable y si su lógica de acción tiene relación con la "Acción Comunicativa" de Habermas. A estos fines se hace una revisión bibliográfica sobre las corrientes teóricas de la innovación tecnológica, desde la perspectiva económica y desde aquellas teorías heredadas de los estudios sociales de la ciencia y la tecnología. Concluyen que no son comunes los abordajes de la teoría de la innovación tecnológica que colocan como condición sine qua non el problema de la sustentabilidad ambiental. Desde esta aproximación destacan que el enfoque de la llamada Ecología Industrial y de los Sistemas de Producción Integrados se distinguen o diferencian de los abordajes económicos por cuanto aquellos se guían no únicamente por los intereses económicos y por el contrario, logran articular las dimensiones económica, social y ambiental de manera indisoluble. Tonelli et. al. (20II) concluyen que si bien es posible que estos sistemas de producción presenten características de sustentabilidad, no pueden afirmar que éstos 
sean orientados por la acción comunicativa al modo Habermasiano, no obstante reconocer su utilidad para el avance en la discusión sobre la perspectiva de la innovación sustentable y el ecodesarrollo. Los autores son conscientes de las limitaciones de este estudio en el sentido de que se basa en constructos teóricos y no en estudios empíricos que aborden directamente la práctica de las personas involucradas en estas nuevas tendencias de producción y las lógicas que dirigen su acción.

El trabajo de Stuchi y Paulino(20II) tiene como objetivo analizar las innovaciones en los servicios públicos, específicamente en los proyectos de reducción de los gases de efecto invernadero desarrollados en los rellenos sanitarios del área metropolitana de la ciudad de Sao Paulo, Brasil, con foco en la aplicación de indicadores de evaluación. Según los autores, el abordaje metodológico de la investigación se basó, por una parte, en el análisis de los multiagentes de innovación, enfatizando en las interacciones de los diversos actores de la esfera política, social y económica capaces de intervenir, directa o indirectamente, en la definición de las peculiaridades del servicio ofertado; y por otra parte, en la adaptación de la Metodología de Carbono Social (MCS), para la construcción de indicadores capaces de medir los resultados sociales y ambientales de los proyectos MDL (Mecanismos de Desarrollo Limpio) ejecutados en los rellenos sanitarios ya mencionados, identificando oportunidades para la innovación en la prestación del servicio relacionado con los residuos sólidos urbanos.

La aplicación del modelo de multiagentes para la innovación en los servicios públicos en el segmento de los residuos sólidos domiciliarios permitió identificar y considerar los actores políticos, las organizaciones de servicios y los usuarios involucrados y se verificó la pertinencia de la aplicación de indicadores definidos a partir de la Metodología del Carbono Social (MCS) para la aprehensión de las peculiaridades del servicio ofertado en el contexto de esta investigación.

El uso irracional de los recursos, así como la generación de contaminantes al medio ambiente producto de emisiones líquidas, gaseosas y sólidas de las industrias son, en muchos casos, consecuencia de la ineficiencia de los procesos y las tecnologías utilizadas al interior de las empresas. Hernández (20lI) plantea que actuando sobre esas ineficiencias es posible el ahorro de materias primas, insumos y energía, mejorando la capacidad competitiva de las empresas y su desempeño ambiental. El objetivo de este trabajo ha sido la utilización de técnicas de producción más limpia (PML) para favorecer la reducción de los impactos ambientales del proceso de producción de la Eritropoyetina humana recombinante (EPO-hr) y sus sistemas auxiliares y así poder cumplir con las normas ambientales establecidas por Cuba. A partir de una evaluación rápida y otra completa de la planta, se identificaron áreas potenciales de aplicación de PML. Los datos obtenidos fueron analizados empleándose una herramienta informática de PML. El uso de esta metodología demostró ser de gran utilidad y su implantación puede contribuir al incremento de los beneficios económicos, posibilitar el acceso a nuevos mercados, mejorar la productividad y competitividad del proceso productivo y además minimizar el riesgo de sanciones de la autoridad ambiental. La identificación de los potenciales de PML a través de la metodología propuesta, posibilitó incrementar la innovación medioambiental del proceso estudiado y se cree que ésta puede ser utilizada en otros procesos biotecnológicos, con características similares al estudiado.

La producción de carbón vegetal con aplicaciones industriales y domésticas es una actividad importante en algunas regiones de Brasil (Rio Grande do Sul), pues genera rentas en pequeños agricultores pero también genera impactos ambientales negativos como son las emisiones de gases a la atmósfera. Estas emisiones pueden ser condensadas y obtenerse un subproducto, el líquido piroleñoso, con aplicaciones en la industria química y en la agricultura, entre otras. El escaso conocimiento de su composición y aplicaciones dificulta su encuadramiento legal y su comercialización. De acuerdo con Dariot, Dewes y Domingues (20I I), el propósito de su estudio ha sido investigar aspectos tecnológicos, económicos y legales para la producción y comercialización del líquido piroleñoso y la posterior transferencia de conocimientos y tecnologías a pequeños productores.

El trabajo de Rivero y Lisboa (20II) tiene como objetivo mostrar, desde una óptica epistemológica, que acciones conscientes que promueven la sustentabilidad se están implantando en la región de la floresta amazónica, específicamente en el municipio de Porto Velho, estado de Rondonia en Brasil. Según los autores, estas acciones no buscan detener el desarrollo económico o afectar la sobrevivencia de los pequeños artesanos que trabajan con materias primas extraídas del bosque amazónico, sino por el contrario, mejorar el bagaje gerencial y técnico de las comunidades organizadas en procura negocios sustentables, de conformidad con las políticas, los procedimientos y la legislación ambiental vigente. Los autores sostienen que frente a los problemas del desempleo en la región, la actividad emprendedora ambiental puede ser una alternativa, teniendo especial cuidado en el manejo sustentable de la selva amazónica. 


\section{Tabla de Contenidos}

ALTEC y la Gestión Tecnológica en Iberoamérica: Gestión de Proyectos, Conocimiento e Innovación Social y Sustentable. Domingo González, Roberto Sbragia, Oscar Galante, Rocío Soto, Renato Valdivieso

Performance Evaluation of IT Projects - The Shenhar and Dvir Model. Renato de Oliveira Moraes, Fernando José Barbin Laurindo

Maturity and Performance in Information Technology Project Management. Renato de Oliveira Moraes, Fernando José Barbin Laurindo

The Cultural Challenges of Managing Global Project Teams: A Study of Brazilian Multinationals. Ivete Rodrigues, Roberto Sbragia

Analysis of the Seven Dimensions of Knowledge Management in Organizations. Camila Lopes Ferreira, Luiz Alberto Pilatti

Understanding the Impact of Project Risk Management on Project Performance: An Empirical Study. Roque Rabechini Junior, Marly Monteiro de Carvalho

Best Practices in Brazilian Companies. Catia dos Reis Machado, Aline França de Abreu, Manoel Agrasso Neto

El Valor de la Tecnología: Enfoques Novedosos para su Determinación. Claudia Nelcy Jimenez, Oscar F. Castellanos

Inter-Organizational Knowledge Conversion and Innovative Capacity in Cooperative Networks. Patricia Trincade Caldas, Gesinaldo Ataide Candido

Test-Driven Development as an Innovation Value Chain. Ana Paula Ress, Renato de Oliveira Moraes, Mário Sérgio Salerno

Comunidades de Práctica - una Innovación en la Gestión del Conocimiento. Edileusa Godói-de-Sousa, Lina Eiko Nakata

Percepción de los Estudiantes de Ingeniería, Tecnología y Curso Técnico sobre Responsabilidad Social Empresarial. Rosângela de Fátima Stankowitz Penteado, Leila Mendes da Luz, Patricio Henrique de Vasconcelos, Hélio Gomes de Carvalho, Antonio Carlos de Francisco

Voices Project: Technological Innovations in Social Inclusion of People with Visual Impairment. Janaina Cazini, Antonio Carlos Frasson 
Online Social Networks and the New Organizational Spaces. Cintia Rodrigues de Oliveira Medeiros, Cristiane Betanho, Jacquelaine Florindo Borges, Rodrigo Miranda, Veronica Angelica Freitas de Paula

Social Enterprises in Brazil: Socially Produced Knowledge Versus Social Innovation. Edileusa Godói-de-Sousa, Valdir Machado Valadão Júnior

Social Technology as a Sustainable Public Policy:The Mandalla Project in Ceará. Josimar Souza Costa, Anna Beatriz Grangeiro Ribeiro Maia, Ana Rita Pinheiro de Freitas, José Carlos Lázaro da Silva Filho, Mônica Cavalcanti Sá Abreu, Marcelo Correia Teixeira Filho

Social Entrepreneurship: A Reflection for Adopting Public Policies that Support the Third Secter in Brazil. Francielli Martins Borges Ladeira, Hilka Vier Machado

Transferencia de Tecnologías a una Cooperativa en Argentina. Un Estudio de Caso. Maximiliano Facundo Vila Seoane, Miguel Leonel Guagliano, Oscar Galante, Antonio Adrián Arciénaga Morales

Services for People Innovation Park - Planning Methodologies. Maria Angela Campelo de Melo, Lygia Magalhães Magacho

Implication of Legal References on Technological Dissemination: A Study on Transgenic Soybeans Resistant to Glyphosate Herbicide in Brazil. Roberta Rodrigues, Celso Luis Salgueiro Lage, Alexandre Guimarães Vasconcellos

Diffusion of Emerging Technologies for Sustainable Development: Prospective Assessment for Public Policies. Maria Fatima Ludovico de Almeida, Carlos Augusto Caldas de Moraes

Awakening the Biodiversity Potential Trough ST\&I Investments in the Sector of Amazonian Biotechnology. Fabiana dos Santos e Souza Frickmann, Alexandre Guimarães Vasconcellos

Strategies for Sustainable Business and Performance in Brazilian Industrial Companies. Clandia Maffini Gomes, Isak Kruglianskas, Flávia Luciane Scherer, Roberto da Luz Neto, Jordana Marques Kneipp

Management of Sustainable Innovation in an Internationalized Company. Uiara Gonçalves De Menezes, Valéria Dias, Clandia Gomes, Flávia Scherer, Isak Kruglianskas

Sustainable Innovation: Eco-development tendencies and Theory of Communicative Action Standpoint. Dany Flávio Tonelli, Elizete Antunes Teixeira, Vânia Aparecida Rezende Oliveira, Gideon Carvalho de Benedicto
$154-165$

$166-176$

$177-187$

$188-196$

197-207

$208-218$

$219-227$

228-238

239-250

264-263

274-284

ISSN: 07 I8-2724. (http://www.jotmi.org)

Journal of Technology Management \& Innovation (c) Universidad Alberto Hurtado, Facultad de Economía y Negocios. 
Public Service Innovation and Assessment Indicators. Silvia Cruz, Sônia Paulino

Producción más Limpia en el Sector Biotecnológico. Yanisleidys Hernández Bermúdez

Vegetable Charcoal and Pyroligneous Acid: Technological, Economical and Legal Aspects of its Production and Commerce. Doriana Daroit,Angela Beatrice Dewes Moura, Igor Paulo Domingues Martins

The Processing and Marketing of Seeds from the Amazon City of Porto Velho, RO. Teresinha Covas Lisboa, Sidney Rivero Tavernard
285-297

298-309

$310-320$

$32|-33|$

\section{Sobre los autores}

Domingo González es doctor en Ingeniería de Producción por la Pontificia Universidade Católica do Rio de Janeiro. Jefe del Departamento de Ingeniería y Director de la Maestría en Gestión y Política de la Innovación y la Tecnología de la Pontificia Universidad Católica del Perú. Presidente de la Asociación Latino-lberoamericana de Gestión Tecnológica (ALTEC).

Roberto Sbragia es graduado, magíster y doctor en administración por la Universidade de São Paulo. Ha realizado un posdoctorado en Management of R\&D por la Northwestern University/Technological Institute/Departament of Industrial Engineering and Management Sciences. Actualmente es profesor titular de la Universidade de São Paulo, en el Departamento de Administración de la FEA, donde coordina el grupo de estudios en Gestión de la Innovación y Proyectos Tecnológicos .Actúa principalmente en los siguientes temas: innovación, evaluación, industria, gestión de la innovación tecnológica y gestión de proyectos.

Oscar Galante, ingeniero de la UNAM- México, y Especialista en Política y Gestión de la Ciencia y la Tecnología de la Universidad del Salvador - Argentina. Investigador en temas de Vinculación Tecnológica. Docente de Posgrado de la UBA, UNGS, UTN, UNSAM, entre otras. A sido Director de Relaciones con la Producción del CONICET, responsable de la Ley de Innovación del FONTAR, Director Nacional de Programas y Proyectos Especiales y Director Nacional de Desarrollo Tecnológico del MinCyT. Actualmente es Gerente de Asistencia Tecnológica para la Demanda Social del INTI; todas instituciones de CyT de Argentina. Miembro de ALTEC, INNRED y REDES. Ex-Presidente de ALTEC en dos períodos.
Rocío Soto es Doctora en Administración y Dirección de Empresas, por la Universidad Politécnica de Cataluña de España. Fue Directora del Centro de Investigaciones Económicas, Administrativas y Sociales del Instituto Politécnico Nacional de México (IPN). Investigadora de la Escuela Superior de Comercio y Administración unidad Sto. Tomás del IPN. Directora por México de ALTEC de octubre de 2009 a la fecha.

Renato Valdivieso es licenciado en Química, Msc. en Planificacion de Ciencia y Tecnología en Cendes-Universidad Central de Venezuela. Es Gerente de Proyectos (Jubilado) de la Fundación Empresas Polar. He sido profesor de posgrado en Cendes-UCV. Es Editor de la Revista Espacios y miembro de la directiva de Altec en los años I99|-I995 y 20II-20I3. 


\section{Referencias Bibliográficas}

ALTEC. (20II). Anales del XIV Congreso Latino-lberoamericano de Gestión Tecnológica. ALTEC, Lima.

ALTEC (2009). Anales del XIII Seminario de la Asociación Latino-lberoamericana de Gestión Tecnológica. Ediciones Tecnológica de Bolívar: Cartagena de Indias.

ALTEC (2008). Anales del XII Seminario de la Asociación Latino-lberoamericana de Gestión Tecnológica. ALTEC, Buenos Aires.

ALTEC (2005). Anales del XI Seminario de la Asociación Latino-lberoamericana de Gestión Tecnológica. ALTEC, Salvador.

ALTEC (2003).Anales del X Seminario de la Asociación Latino-lberoamericana de Gestión Tecnológica. ALTEC, México D.F.

ALTEC (200I). Anales del IX Seminario de la Asociación Latino-lberoamericana de Gestión Tecnológica. ALTEC, San José.

ALTEC (1999). Anales del VIII Seminario de la Asociación Latino-lberoamericana de Gestión Tecnológica. ALTEC,Valencia.

ALTEC (1997). Anales del VIII Seminario de la Asociación Latino-lberoamericana de Gestión Tecnológica. ALTEC, La Habana.

ALTEC (1995). Anales del VI Seminario de la Asociación Latino-Iberoamericana de Gestión Tecnológica. ALTEC, Concepción.

ALTEC (1993).Anales delV Seminario de la Asociación Latino-lberoamericana de Gestión Tecnológica. ALTEC, Bogotá.

ALTEC (|99|). Anales del IV Seminario de la Asociación Latino-lberoamericana de Gestión Tecnológica. ALTEC, Caracas.

ALTEC (1989). Anales del III Seminario de la Asociación Latino-lberoamericana de Gestión Tecnológica. ALTEC, Buenos Aires.

ALTEC (1987).Anales del II Seminario de la Asociación Latino-lberoamericana de Gestión Tecnológica. ALTEC, México D.F.
ALTEC (1985). Anales del I Seminario de la Asociación Latino-lberoamericana de Gestión Tecnológica. ALTEC, São Paulo.

Galante, O. y Perez, N. (2008). Reseña Histórica de la Asociación Latino-iberoamericana de Gestión Tecnológica. ALTEC, Buenos Aires.

Quirino T., Baia, M., Rodrigues, I. y Sbragia, R. (200I). Innovación tecnológica, su política y gestión en el mundo ibérico: Cómo se refleja la disciplina en el Congreso de la ALTEC. Espacios.Vol. 22 (3).

Sbragia, R., Rodrigues, I. Baiao, M., Rego, T. (2003). Política e Gestão da Inovação Tecnológica: Estudo Comparativo da Evolução da Disciplina no Brasil e na Ibero-América. Anales del X Seminario de la Asociación Latino-lberoamericana de Gestión Tecnológica.ALTEC, México D.F. 
J.Technol. Manag. Innov. 2013,Volume 8, Special Issue ALTEC. 